\title{
Síndrome de la orina rosa
}

\section{Pink urine syndrome}

\author{
Luis Del Carpio-Orantes* y Leonora Beltrán-Jiménez
}

Departamento de Medicina Interna, Hospital General de Zona 71, Delegación Veracruz Norte, Instituto Mexicano del Seguro Social. Veracruz, México

\begin{abstract}
Resumen
Introducción: El síndrome de la orina rosa se caracteriza por ser una entidad clínica benigna y de curso autolimitado, a menudo sin requerir intervención terapéutica, cuya característica es la tinción de la orina de un color rosa tenue, asociada a la precipitación de cristales de ácido úrico. Tiene como factores predisponentes el uso de propofol (entre otros fármacos), así como sobrepeso/obesidad, neoplasias, cirugía, entre otras. Siendo un síndrome de baja incidencia, benigno y relacionado a cuestiones metabólicas, cáncer, cirugías y fármacos, consideramos importante su comunicación. Material y métodos: Se revisan los casos de síndrome de la orina rosa documentados en el Hospital General de Zona 71 de la Ciudad y Puerto de Veracruz, México, durante el periodo de 2017 a 2018. Se caracterizan acorde a la etiología, factores predisponentes y evolución. Resultados: Se reportan 3 casos del síndrome de la orina rosa. El género más afectado fue el masculino, los factores predisponentes fueron sobrepeso, cáncer, cirugía y el uso de propofol en infusión. En todos se documentó cristaluria de ácido úrico, que confiere el color rosa característico de este síndrome. La resolución fue espontánea en todos los casos en el plazo de 1 a 2 horas. Conclusiones: El síndrome de la orina rosa, pese a ser una entidad benigna y autolimitada, ofrece una amplia gama de condiciones que actúan como predisponentes, como síndrome metabólico, cáncer, cirugías y diversos fármacos que podrían favorecer cada vez más su aparición, por lo que es una entidad a reconocer por los clínicos como indicador de cristaluria de ácido úrico, de igual forma en el contexto de enfermedades metabólicas.
\end{abstract}

Palabras clave: Propofol. Cáncer. Ácido úrico. Orina rosa.

\section{Abstract}

Introduction: The pink urine syndrome is characterized by being a benign clinical entity and of course self-limited often without requiring therapeutic intervention, whose characteristic is the staining of the urine of a faint pink color, associated with the precipitation of uric acid crystals, having as predisposing factors the use of propofol (among other drugs), as well as overweight/obesity, malignancies, surgery, among others. Being a low incidence syndrome, benign and related to metabolic issues, cancer, surgery and drugs, we consider its communication important. Material and methods: The cases of pink urine syndrome documented in the General Hospital of Zone 71 of the City and Port of Veracruz, Mexico, are reviewed during the period from 2017 to 2018. They are characterized according to the etiology, predisposing factors and evolution. Results: 3 cases of pink urine syndrome are reported, the most affected gender was male, the predisposing factors were: overweight, cancer, surgery and the use of propofol in infusion. In all, uric acid crystalluria was documented, which gives it the characteristic pink color of this syndrome. The resolution was spontaneous in all cases within 1-2 $\mathrm{h}$. Conclusions: The pink urine syndrome, despite being a benign and self-limited entity, offers a wide range of conditions that act as predisposing factors such as

Correspondencia:

*Luis Del Carpio-Orantes

E-mail: neurona23@ hotmail.com
Disponible en internet: 10-03-2020 Rev Hosp Jua Mex. 2020;87(1):39-42

www.revistahospitaljuarez.com 1405-9622/@ 2019 Sociedad Médico-Quirúrgica del Hospital Juárez de México, A.C. Publicado por Permanyer. Este es un artículo open access bajo la licencia CC BY-NC-ND (http://creativecommons.org/licenses/by-nc-nd/4.0/). 
metabolic syndrome, cancer, surgeries and various drugs that could increasingly favor its appearance, being an entity to be recognized by clinicians as an indicator of uric acid crystalluria in the same way in the context of metabolic diseases.

Key words: Propofol. Cancer. Uric acid. Pink urine.

\section{Introducción}

El síndrome de la orina rosa se caracteriza por la excreción súbita y aguda de orina de color rosa, distinguible fácilmente de la hematuria por su color más tenue y por dejar sedimento urinario de dicho color.

El mecanismo por el cual se desarrolla este síndrome es por incremento en la tasa de excreción de uratos o ácido úrico, lo cual le da la coloración característica a la orina, favorecido por un aumento de la liberación de hormona antidiurética y/o cortisona. Recientemente se ha implicado la activación de la vía hemooxigenasa-1 (que se produce normalmente durante el estrés oxidativo y protege de daño celular) por estimulo de propofol o asociado a la presencia de resistencia a la insulina ${ }^{1-3}$.

Las causas más frecuentes son el empleo de fármacos con propiedades uricosúricas (fármacos que aumentan la excreción de ácido úrico en la orina y disminuyen los niveles de uricemia), y los principales reportados son: propofol, probenecid, losartán, amlodipino, guaifenesina, fenofibrato y atorvastatina. De igual forma, otras condiciones pueden condicionar un efecto uricosúrico, como las cirugías abdominales y la presencia de tumores o cáncer. Asimismo, los pacientes con síndrome metabólico u obesidad, así como aquellos con hiperuricemia o gota, pueden presentar síndrome de la orina rosa por incremento en la tasa de excreción metabólica de uratos ${ }^{4-8}$.

Otros condiciones propios de pacientes críticamente enfermos, como deshidratación, acidosis metabólica, oliguria, aciduria e hiperosmolaridad urinaria, condicionan también un efecto uricosúrico, lo que condiciona el síndrome ${ }^{9}$.

Se considera una manifestación benigna que no interfiere con la tasa de filtrado glomerular y que no requiere de un tratamiento específico, sino observación, ya que tiende a desaparecer de forma paulatina.

Recientemente se publicó una revisión no exhaustiva de casos, completando 14 casos, aunque no se tienen datos uniformes (no todos tienen índice de masa corporal [IMC], niveles de ácido úrico, etc.), se aprecian las siguientes características: 6 casos son de pacientes asiáticos, los demás dispersos en América y Europa; 11 casos son de pacientes del género masculino; el grupo etáreo más afectado es el de 61 a 70 años; 3 casos cursaron con obesidad, sin embargo, gran parte de la cohorte no tiene registrado el dato de peso/talla/ IMC, de igual forma, solo 7 casos tienen registrados los niveles de ácido úrico, y solo 2 de ellos cursan con hiperuricemia; en forma destacable, 9 casos emplearon propofol en forma previa al inicio de la manifestación de orina rosa; solo 2 casos presentaron lesión renal aguda; 3 casos estuvieron asociados a neoplasias; y 5 casos se asociaron a cirugías diversas ${ }^{10}$.

Consideramos importante la comunicación de estos casos que, pese a su benignidad, pueden indicar alteraciones metabólicas asociadas a síndrome metabólico o cáncer, siendo favorecidas por el efecto farmacológico de ciertos medicamentos, destacando el propofol.

\section{Material y métodos}

Se reportan los casos documentados de síndrome de la orina rosa presentados en el Hospital General de Zona 71 de la delegación Veracruz Norte del Instituto Mexicano del Seguro Social, de 2017 a 2019. Fueron tres los casos, cuyas características se aprecian en la tabla 1 y las figuras 1 a 3 .

Estos pacientes ameritaron estancia en la Unidad de Cuidados Intensivos dadas sus características nosológicas, presentaron el síndrome de la orina rosa entre la primera y segunda hora de estancia y tras la exposición parenteral a propofol. Siempre se documentó la presencia de cristales de ácido úrico en la orina, que a la clínica tenía un franco color rosa y, en dos casos, franco sedimento urinario de dicho color, sin embargo, el curso fue benigno y fugaz, y desapareció a las pocas horas de forma espontánea, sin necesidad de intervenciones especiales, por lo que el síndrome es una manifestación transitoria sin recurrencia y que no condiciona una lesión renal o de otro tipo. Los factores predisponentes identificados fueron sobrepeso y obesidad (sin cumplir criterios de síndrome metabólico), así como cirugía y neoplasias.

\section{Discusión}

Según la revisión documentada de los casos a nivel internacional, casi todos los casos se asocian a la administración de fármacos, y se resalta que más del $70 \%$ se asocian a la administración de propofol. Como factores predisponentes se encuentran, en la bibliografía, la 
Tabla 1. Características clínicas y bioquímicas de los casos con síndrome de la orina rosa

\begin{tabular}{|c|c|c|c|}
\hline & Caso 1 & Caso 2 & Caso 3 \\
\hline Edad & 30 años & 50 años & 45 años \\
\hline Género & Masculino & Femenino & Masculino \\
\hline Antecedentes & Negados & Hipertensión arterial & Obesidad \\
\hline $\begin{array}{l}\text { Índice de masa } \\
\text { muscular }\end{array}$ & $29 \mathrm{~kg} / \mathrm{m}^{2}$ (sobrepeso) & $24 \mathrm{~kg} / \mathrm{m}^{2}$ (normal) & $40.2 \mathrm{~kg} / \mathrm{m}^{2}$ (obesidad III) \\
\hline Diagnóstico actual & $\begin{array}{l}\text { Politrauma } \\
\text { Traumatismo craneal severo } \\
\text { Cirugía craneal } \\
\text { Muerte cerebral }\end{array}$ & $\begin{array}{l}\text { Cirugía de resección de } \\
\text { meningioma }\end{array}$ & $\begin{array}{l}\text { Adenocarcinoma esofágico no metastásico } \\
\text { Esofaguectomía }\end{array}$ \\
\hline Medicación prescrita & Propofol & Propofol & Propofol \\
\hline Dosis inicial & $1.5 \mathrm{mg} / \mathrm{kg}$ & $1.5 \mathrm{mg} / \mathrm{kg}$ & $2 \mathrm{mg} / \mathrm{kg}$ \\
\hline Dosis de mantenimiento & $200 \mathrm{mcg} / \mathrm{kg} / \mathrm{min}$ & $200 \mathrm{mcg} / \mathrm{kg} / \mathrm{min}$ & $200 \mathrm{mcg} / \mathrm{kg} / \mathrm{min}$ \\
\hline $\begin{array}{l}\text { Aparición de orina rosa } \\
\text { posterior al inicio de la } \\
\text { sedación }\end{array}$ & 2 horas & 1 hora & 1 hora \\
\hline Hallazgos de laboratorio & $\begin{array}{l}\text { Cristaluria de ácido úrico } \\
\text { Ácido úrico: } 5.7 \mathrm{mg} / \mathrm{dl}\end{array}$ & $\begin{array}{l}\text { Cristaluria de ácido úrico } \\
\text { Ácido úrico: } 6.2 \mathrm{mg} / \mathrm{dl}\end{array}$ & $\begin{array}{l}\text { Cristaluria de ácido úrico } \\
\text { Ácido úrico: } 7.1 \mathrm{mg} / \mathrm{dl}\end{array}$ \\
\hline Lesión renal aguda & No & No & No \\
\hline Síndrome metabólico & No & No & No \\
\hline
\end{tabular}

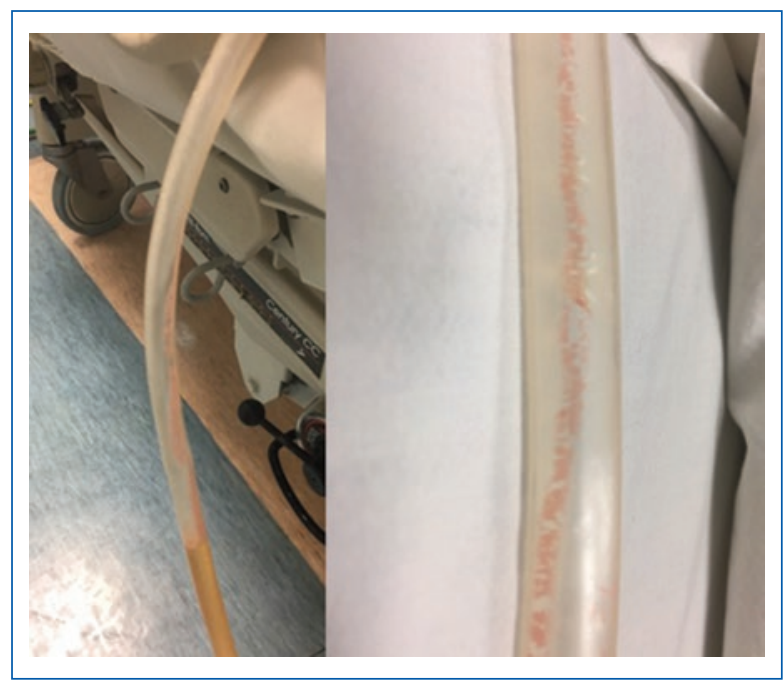

Figura 1. Hombre de 30 años, orina rosa y sedimento urinario rosa. El uroanálisis reporta cristaluria de ácido úrico.

presencia de cáncer y cirugías, aunado a baja incidencia de sobrepeso/obesidad e hiperuricemia, situaciones que se reprodujeron en los casos que presentamos, donde destaca también la administración de propofol y factores predisponentes como cáncer y sobrepeso, y como factores precipitantes cirugía y la aplicación del

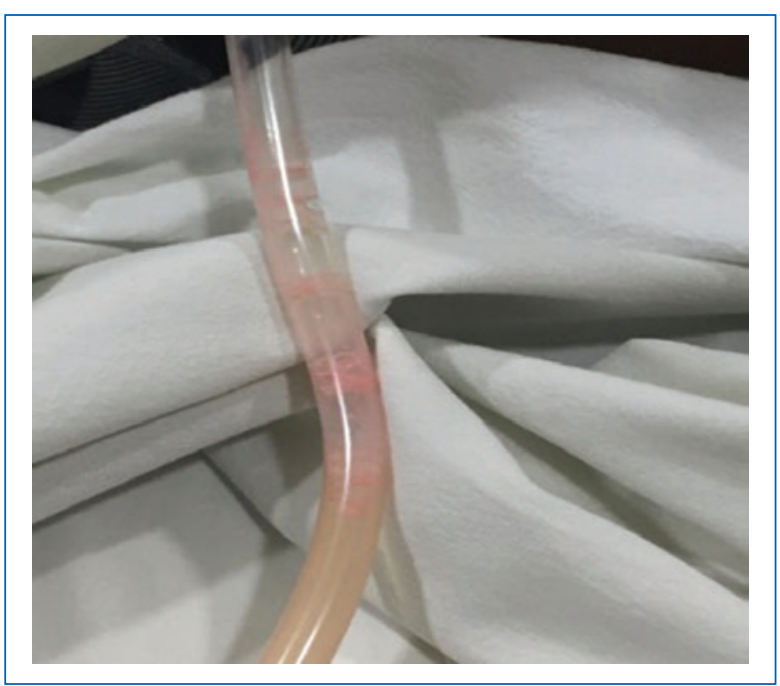

Figura 2. Mujer de 50 años edad, franca orina rosa sin sedimento. El uroanálisis reporta cristales de ácido úrico.

fármaco, por lo que los datos parecen ser concordantes en forma fehaciente. En la serie internacional se menciona la presencia de lesión renal aguda, que en nuestros casos no se ha documentado, probablemente asociada a otras situaciones distintas, como la precipitación de cristales de ácido úrico en los túbulos renales 


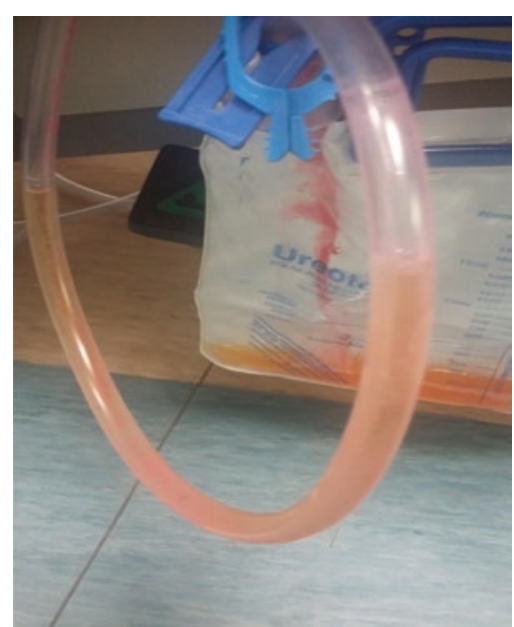

Figura 3. Hombre de 45 años de edad, franca orina rosa con abundante sedimento urinario rosa. Se demostró cristaluria de ácido úrico.

en el contexto de un paciente con hiperuricemia, situación que provoca insuficiencia renal crónica ${ }^{10}$.

\section{Conclusiones}

El síndrome de la orina rosa, pese a ser un síndrome benigno y autolimitado que no requiere mayor intervención terapéutica, es importante identificarlo y conocer su patogénesis, ya que su presencia puede hablar de alteraciones relacionadas al metabolismo del ácido úrico, el cual se asocia fuertemente a síndrome metabólico, cáncer o cirugías, y que puede ser favorecido por la exposición a fármacos como propofol o la realización de cirugías.

\section{Conflicto de intereses}

Los autores declaran no tener conflicto de intereses alguno.

\section{Financiamiento}

Los autores no recibieron patrocinio para llevar a cabo este artículo.

\section{Responsabilidades éticas}

Protección de personas y animales. Los autores declaran que los procedimientos seguidos se conformaron a las normas éticas del comité de experimentación humana responsable y de acuerdo con la Asociación Médica Mundial y la Declaración de Helsinki.

Confidencialidad de los datos. Los autores declaran que han seguido los protocolos de su centro de trabajo sobre la publicación de datos de pacientes.

Derecho a la privacidad y consentimiento informado. Los autores han obtenido el consentimiento informado de los pacientes y/o sujetos refereridos en el artículo.

\section{Bibliografía}

1. Dinis-Oliveira RJ. Metabolic Profiles of Propofol and Fospropofol: Clinical and Forensic Interpretative Aspects. BioMed Research International. 2018;2018:6852857.

2. Stern $A B$, Stewart HD, Singh HK, Kshirsagar AV. Pink urine after propofol anesthesia. Kidney International. 2010;78(11):1193.

3. Del Carpio-Orantes L, Elizalde-Bernal RJJ. Villegas-Dominguez JE, Morales-Ocon JE, Tejero Da Silveira RK. Pink urine syndrome. AMJ. 2017;10(3):199-201.

4. Del Carpio-Orantes L. Propofol. Pink Urine Syndrome. Case report. Reactions Weekly. 2017;1654:295.

5. Potton L, Bonadona A, Minet C, Timist JF. Pink urine. Intensive Care Medicine. 2013;39(3):389-90.

6. Okubo K, Okubo H, Kamijo Y, Higuchi M. Pink Urine Syndrome. Internal Medicine. 2011:50(18):2057.

7. Ogawa S, Takiguchi J, Shimizu M, Nako K, Okamura M, Kinouchi Y, et al. The Reduction in Urinary Glutamate Excretion Is Responsible for Lowering Urinary $\mathrm{pH}$ in Pink Urine Syndrome. Tohoku J Exp Med. 2016;239(2):103-10

8. Viswanathan S. Urine bag as a modern day matula. ISRN Nephrology. 2013;2013:215690.

9. Thamcharoen N, Leeaphorn N, Sanguankeo A. A pretty clue in pink: pink urine in a morbidly obese patient with lymphoma. Clinical Nephrology. 2015;84(8):116.

10. Tucker, Bryan M. \& Perazella Mark A. Pink Urine Syndrome: A Combination of Insulin Resistance and Propofol. Kidney Int Rep. 2019; $4(1): 30-9$. 